\title{
La inmunización y los servicios relacionados con el embarazo en Guatemala*
}

\author{
Anne R. Pebley* * \\ Noreen Goldman * *
}

En este artículo se examina la experiencia de Guatemala en la provisión de inmunización infantil, hecha parcialmente por una campaña nacional de vacunación, y en la provisión de servicios de salud relacionados con el embarazo por un sistema de entidades gubernamentales y no gubernamentales, durante los años ochenta. Los resultados muestran que los programas de salud basados predominantemente en campañas alcanzan con frecuencia a más sectores de la población en comparación a los servicios provistos por clínicas. Específicamente, las diferencias en el uso de servicios relacionados con el embarazo son más grandes por etnicidad individual, etnicidad de la comunidad, distancia de una clínica, y distancia de la ciudad de Guatemala, en comparación a la cobertura del programa de vacunación.

Durante los años ochenta los programas de sobrevivencia infantil dominaron las actividades de salud pública en un número considerable de países en vías de desarrollo. Estos programas se concentraron generalmente en estrategias sencillas, cuya selección se basó en lograr el mayor impacto sobre la salud infantil al menor costo (Pebley, 1993; Warren, 1988). Uno de los elementos más exitosos de estos programas es la campaña de inmunización infantil (Gadmoski y Black, 1990). Una ventaja importante de este programa es que típicamente no depende de la existencia o eficacia de una clínica local.

* Las autoras agradecen el apoyo para esta investigación a NIcHD (National Institute of Child Health and Human Development) (beca número R01 HD27361) y a la NSF (National Science Foundation) (beca número BNS-8700864). Este trabajo fue llevado a cabo en el Centro de Estudios Avanzados sobre las Ciencias del Comportamiento, en Stanford, en el Departamento de Demografía de la Universidad de California en Berkeley, en la Oficina de Estudios sobre la Población en la Universidad de Princeton, y en el Instituto de Nutrición de Centroamérica y Panamá en Guatemala; cada uno de ellos proporcionó generosamente sus servicios y asistencia a las autoras.

Las opiniones expresadas en este trabajo, sin embargo, no necesariamente reflejan la opinión de estas organizaciones. Las autoras agradecen a los doctores Lorenzo Moreno, Germán Rodríguez, Elena Hurtado, Sheila Cosminsky y Jorge Hermida Córdova por sus sugerencias a una versión anterior de este documento, y a la señora Rachel Veerman por su excelente asistencia con la traducción y producción del artículo.

** Population Research Center, Rand.

*** Office of Population Research, Princeton University. 
Preocupaciones sobre la salud de la mujer durante los años ochenta (Mahler, 1987; Kwast, 1991) y la evidencia creciente de la importancia del control prenatal y la atención del parto para la salud maternal e infantil (Institute of Medicine, 1985), han generado un nuevo interés sobre el mejoramiento de los servicios durante el embarazo y el parto. Por ejemplo, el objetivo de la Cumbre Mundial para la Niñez de 1990 fue añadir al programa básico de sobrevivencia infantil el "acceso al control prenatal, una partera adiestrada durante el parto y un sistema de referencia para embarazos de alto riesgo y emergencias obstétricas" para todas las mujeres (Grant, 1993: 59). Como se describe en este trabajo, basado en la experiencia de Guatemala en los años ochenta, la provisión equitativa de servicios relacionados con el embarazo en países poco industrializados involucra asuntos operacionales, sociales y culturales que, en su enfoque y complejidad, supera a los enfrentados en las campañas de inmunización y a la mayoría de las intervenciones asociadas con los programas de sobrevivencia infantil.

En este artículo se examina la experiencia de Guatemala en la provisión de inmunización infantil, hecha parcialmente por una campaña nacional de vacunación, y en la provisión de servicios de salud relacionados con el embarazo por un sistema de entidades gubernamentales y no gubernamentales, durante los años ochenta. Específicamente, se hace una comparación entre la cobertura de estos dos tipos de servicios basada en datos de una encuesta nacional realizada en 1987. La hipótesis central es que el alcance del sistema de salud pública guatemalteca en relación con servicios para mujeres embarazadas fue muy limitado en comparación con el programa de inmunización y, específicamente, que el programa de inmunización fue mucho más exitoso en alcanzar a la población indígena, pobre y residentes de áreas lejanas de la ciudad capital. El objetivo central es entender el tamaño de la tarea involucrada en la extensión de servicios de salud fuera de las estrategias de los programas de sobrevivencia infantil como la inmunización, hasta servicios más complejos como los relacionados con el embarazo.

\section{Método}

En Guatemala, los servicios de salud públicos y privados para mujeres embarazadas son proporcionados principalmente por médicos, enfermeras y auxiliares. Sin embargo, como en muchos otros países (Parra, 1991; Isenalumbe, 1990), parteras tradicionales aún proporcionan la mayoría de los servicios relacionados con el embarazo. A pesar de existir un programa para el adiestramiento de 
parteras tradicionales, la mayoría de éstas no tienen entrenamiento formal ni están afiliadas a ningún servicio de salud formal (Bossert y Del Cid Peralta, 1987). En contraste, la inmunización contra enfermedades infantiles es proporcionada exclusivamente por el sistema de salud público y médicos privados, y no por proveedores tradicionales. Las inmunizaciones fueron proporcionadas en forma limitada al principio de los años noventa. En 1986, el nuevo gobierno civil emprendió una importante campaña de inmunización contra las enfermedades infantiles (difteria, tosferina, tétano, sarampión y polio). Fue considerado un éxito importante (MSPAs, 1987), aunque quizá este esfuerzo recanalizó recursos de servicios regulares de salud. No hubo campaña de vacunación en 1987, pero éstas se han conducido regularmente en los años subsiguientes (Goldman y Pebley, 1994). Inmunizaciones y otros servicios de salud del gobierno son proporcionados gratuitamente.

La utilización de los servicios de salud difiere considerablemente entre los dos grupos étnicos, cada uno de los cuales aproximadamente integra la mitad de la población (Warren et al., 1987; MSPAs y otros, 1989). La población indígena (descendientes de los mayas y otros grupos precolombinos) conserva una identidad separada y lenguas indígenas. Los servicios médicos y el sistema de salud pública son menos utilizados por los indígenas que por la otra mitad de la población, conocida como ladina, quien habla únicamente español, se viste con ropa europea y se identifica con la cultura nacional guatemalteca. Es más común que la población indígena viva en áreas rurales y en partes remotas del país, y además son más pobres y poseen un menor nivel de escolaridad que los ladinos. Un estudio realizado en 1986 encontró que las áreas de residencia con la menor cantidad de personal de salud e instalaciones sanitarias en el sector formal fueron predominantemente indígenas, así como también las más lejanas de la capital (Von Hoegen, 1986).

\section{Datos}

Los datos de Guatemala para este análisis fueron tomados de la Encuesta Nacional de Salud Materna Infantil (ENSMI) conducida en 1987 (MSPAs y otros, 1989) en Guatemala. Esta encuesta se basó en una muestra nacional de 5160 mujeres de 15 a 44 años de edad, entrevistadas entre los meses de septiembre y diciembre de 1987. La entrevista incluyó preguntas sobre el control prenatal y atención del parto de todos los 4627 nacidos vivos durante los cinco años previos a la entrevista, así como preguntas sobre el estado de inmunización de los 4230 infantes nacidos durante este periodo y 
de sobrevivientes al momento de la entrevista. Los datos no nos permiten distinguir entre parteras adiestradas y otras; sin embargo, como señalamos con anterioridad, la mayoría de las parteras no tienen adiestramiento formal. Además, solamente el proveedor "más calificado" que proporcionó el control prenatal y la atención del parto fue codificado para cada embarazo, sin tener en cuenta cuántos tipos diferentes de proveedores fueron consultados.

La información sobre ocho inmunizaciones infantiles fue recopilada para todos los niños menores de cinco años al momento de la entrevista: tres dosis de DPT, tres dosis de la vacuna de polio, una dosis de BCG (antituberculosis) y una dosis de la vacunación contra el sarampión. La información se copió de los carnets de vacunación y fue complementada con reportes maternales. En un trabajo anterior (Goldman y Pebley, 1994), se muestra que al incluir información provista por la madre en los cálculos sobre la provisión de inmunizaciones, la precisión de nuestras estimaciones mejoran considerablemente. Por lo tanto, los resultados aquí presentados incorporan ambos tipos de datos. La ENSMI también proporciona información sobre la distancia a la clínica más cercana (por ejemplo, los centros de salud del gobierno, un servicio de una organización privada voluntaria o una clínica privada) para aproximadamente $66 \%$ de los conglomerados en los cuales la encuesta fue levantada. Los resultados de estos conglomerados no aparentan tener diferencias sustanciales en comparación a todos los conglomerados en que la encuesta fue realizada (Pebley y Goldman, 1992). La proporción de personas dentro de cada localidad reportadas como indígenas fueron tomadas de datos a nivel municipal del censo de 1981.

A menos que se indique lo contrario, las muestras consideradas en este análisis consisten en embarazos que terminaron con nacimientos vivos durante los 5 años anteriores a la entrevista en los conglomerados incluidos en la muestra, para los que la información sobre la distancia a la clínica más cercana está disponible $(n=3467)$. Todos los análisis referentes a la inmunización están basados en una segunda muestra de infantes entre uno a cinco años de edad ( $n=2495)$ de la entrevista. Los infantes menores de un año fueron excluidos porque aún no habían alcanzado la edad adecuada para ser provistos de algunas inmunizaciones.

\section{Análisis}

El análisis descriptivo (véase el cuadro 1) muestra el porcentaje de embarazos y partos que fueron atendidos según tres fuentes 


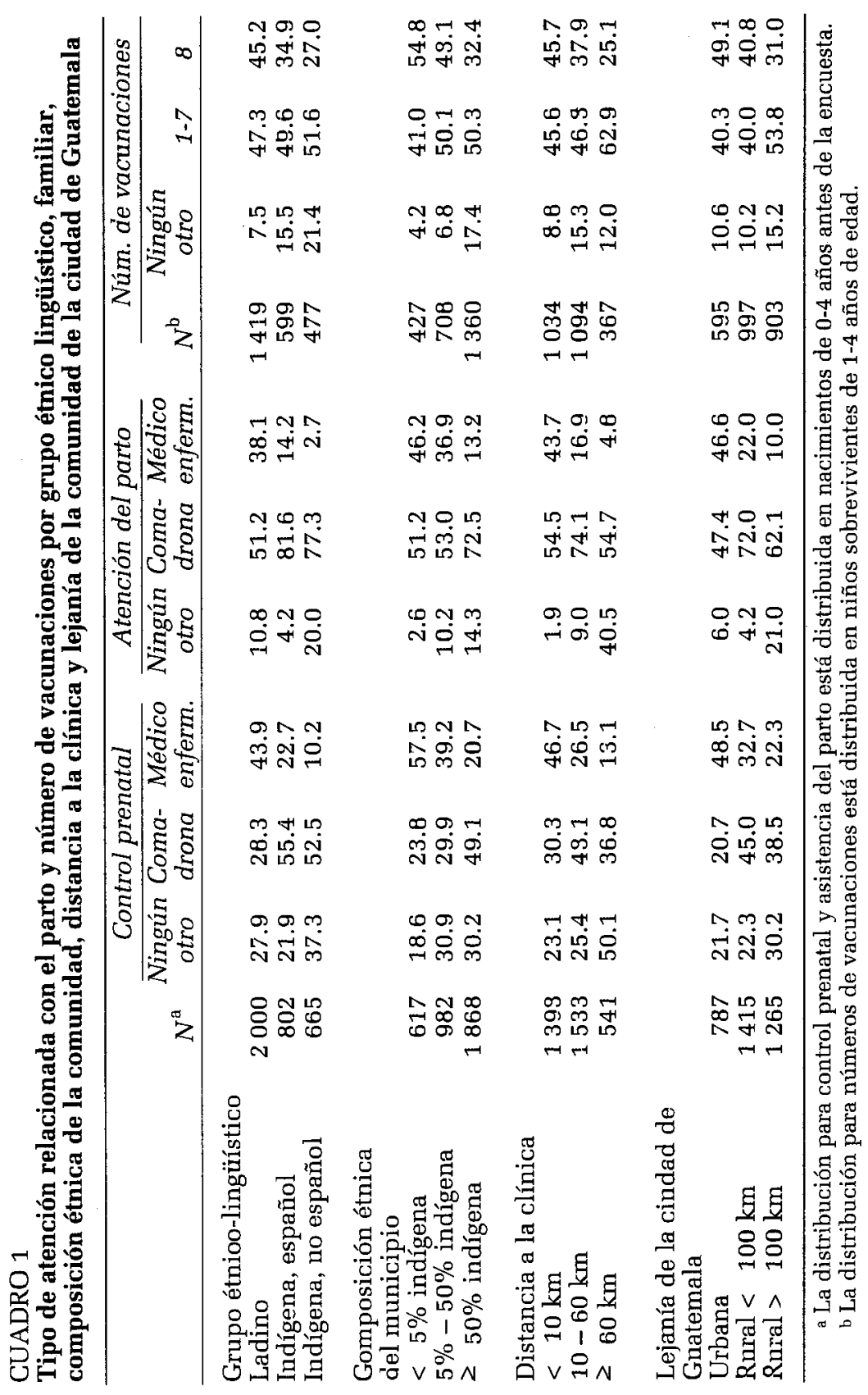


posibles: 1) personal no profesional o ningún cuidado; 2) parteras o comadronas, y 3) médicos o enfermeras. Médicos y enfermeras se agrupan en solamente una categoría en este análisis debido a que proporcionan tipos similares de servicios en Guatemala, aunque la provisión de servicios por los médicos es mucho más frecuente. Las distribuciones se presentan separadamente para los grupos etnolingüísticos, al igual que para las categorías que indican la composición étnica de la municipalidad, la distancia de la comunidad a la clínica más cercana y la lejanía de la comunidad de la ciudad de Guatemala. También se indica el número de vacunas $(0,1-7$ u 8) reportadas o registradas en los carnets de vacunación de un máximo de ocho inmunizaciones recomendadas por el Ministerio de Salud Pública de Guatemala. Siete de las ocho inmunizaciones fueron ofrecidas por la campaña de inmunización de 1986, con excepción de la antituberculosis. Aproximadamente la mitad de las inmunizaciones a los niños incluidos en la muestra fueron provistas durante la campaña de 1986 (Goldman y Pebley, 1994). El análisis de inmunización se basó en una submuestra de la muestra total de sobrevivientes y que tenían al menos un año de edad al momento de la entrevista. La restricción de las estimaciones de inmunización a niños vivos resultó probablemente en una pequeña sobreestimación de la cobertura de inmunización, dado que los resultados de otras encuestas sugieren que la posibilidad de ser vacunados es más baja entre los niños que mueren durante la infancia que entre los niños sobrevivientes al momento de la entrevista.

Las estimaciones del cuadro 2 reflejan la relación entre las diferentes formas de contacto con servicios biomédicos de salud para los tres grupos etnolingüísticos. Los servicios biomédicos se definen en este análisis como la consulta de un doctor o enfermera para el control prenatal, atención del parto o haber recibido todas las inmunizaciones. La correlación de Pearson muestra la relación del control biomédico de salud con otro tipo de servicios entre los individuos de cada uno de los tres grupos etnolingüísticos.

Una regresión logística multivariada fue utilizada para determinar el efecto de factores étnicos y de residencia sobre el uso de servicios biomédicos de salud controlando las siguientes variables: edad del niño, edad de la madre, orden del nacimiento del niño, nivel de escolaridad de la madre, situación de empleo de la madre, nivel de escolaridad del esposo, profesión u ocupación del esposo y la posesión de un televisor, así como la frecuencia con la que se le ve.

El uso de controles es un componente esencial de este análisis, puesto que las características demográficas y socioeconómicas de 
CUADRO 2

Relación entre tres formas de servicios biomédicos de salud para un dado embarazo/niño por grupo étnico-lingüístico*

\begin{tabular}{|c|c|c|c|c|}
\hline & Total & $\begin{array}{c}\text { Indígena } \\
\text { No español }\end{array}$ & $\begin{array}{l}\text { Indígena } \\
\text { Español }\end{array}$ & Ladino \\
\hline Número de hijos & 3305 & 670 & 758 & 1877 \\
\hline $\begin{array}{l}\text { Correlación, control prenatal } \\
\text { y atención del parto }\end{array}$ & 0.55 & 0.22 & 0.46 & 0.51 \\
\hline e inmunización & 0.22 & 0.12 & 0.11 & 0.20 \\
\hline $\begin{array}{l}\text { Correlación, atención del parto } \\
\text { e inmunización }\end{array}$ & 0.21 & 0.00 & 0.07 & 0.19 \\
\hline
\end{tabular}

* Limitado a hijos sobrevivientes de 1-4 años de edad.

los infantes y sus familias están probablemente relacionadas tanto con la utilización de los servicios biomédicos de salud así como con las variables étnicas y geográficas. Los resultados de los modelos logísticos son presentados como razones de chanzas (por ejemplo, las chanzas ${ }^{1}$ de recibir servicios biomédicos en relación con las chanzas de recibir un servicio alternativo). La primera serie de columnas en el cuadro 3 muestra la razón de chanzas ${ }^{2}$ asociada con factores étnicos y de localidad de residencia, en la ausencia de cualquier variable de control; la segunda serie de columnas muestra razones de chanzas correspondientes al caso donde se han incluido todas las variables de control reportadas anteriormente. Se calculan estas razones de chanzas por exponenciación de los coeficientes respectivos en el modelo logístico. Cada razón de chanzas se calcula con relación a la categoría omitida para esa variable. Por ejemplo, en la primera columna del cuadro, la razón de chanzas estimada de 0.15 indica que las chanzas de recibir atención prenatal biomédica para un niño indígena de una familia qme no habla español tiene aproximadamente un séptimo de las chanzas que de un niño ladino (dado que "ladino" es la categoría omitida).

1 Chanzas y probabilidades tienen la siguiente diferencia: un ejemplo de una probabilidad es el número de partos asistidos por un médico, dividido por el número de partos totales. La chanza correspondiente sería el número de partos asistidos por un médico, dividido por el número de partos no asistidos por un médico. Una razón de chanzas se refiere a la chanza asociada con una categoría de una variable independiente en comparación con la chanza de la categoría omitida.

$2 \mathrm{O}$ sea, odds ratic. 


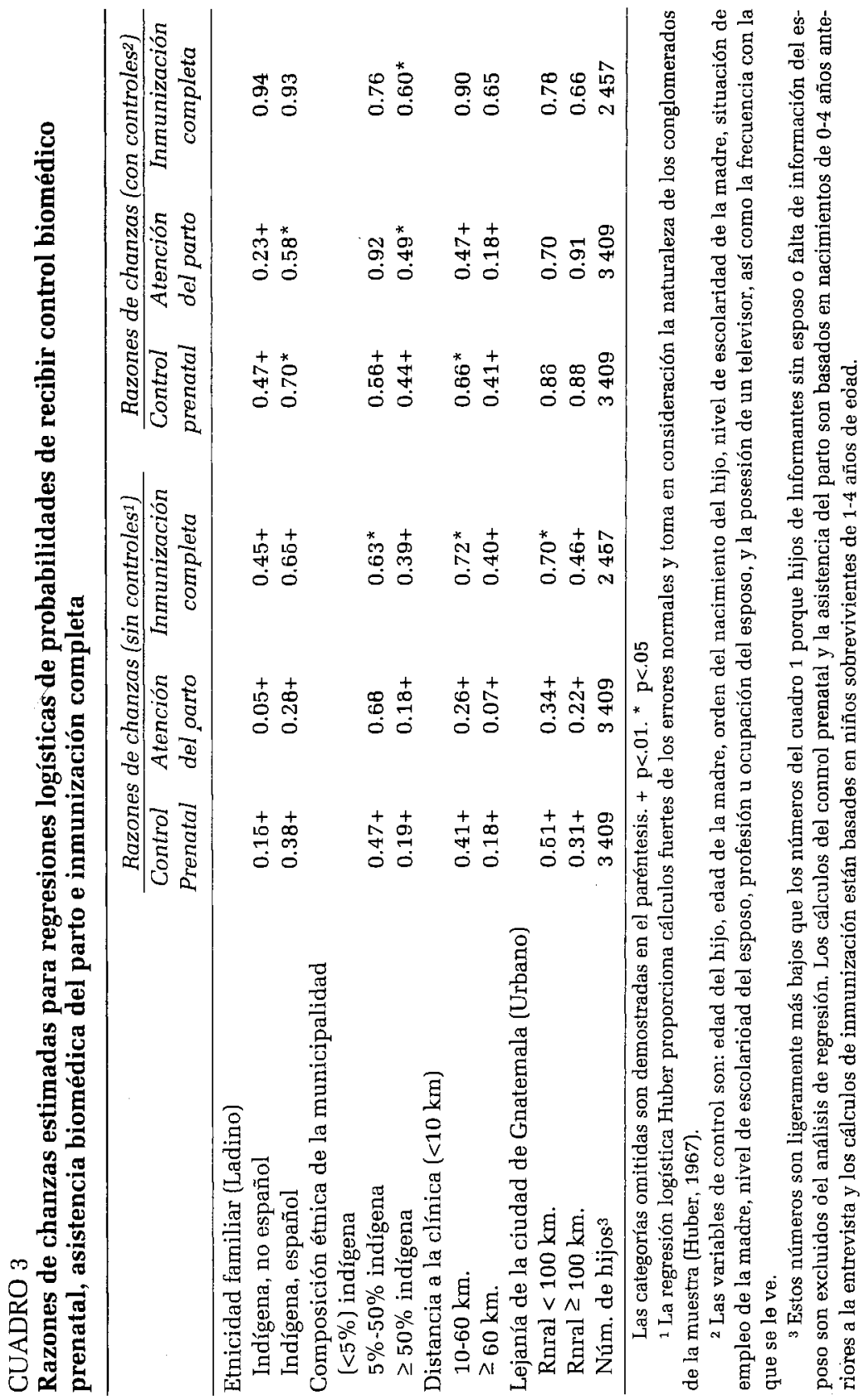




\section{Resultados}

Las proporciones del cuadro 1 muestran las grandes variaciones por etnicidad y localidad de residencia en la consulta de médicos y enfermeras para el control prenatal y atención del parto. Por ejemplo, es 14 veces más probable que las mujeres ladinas consulten a un médico o a una enfermera al momento del parto que mujeres indígenas. La residencia en una comunidad predominantemente indígena, en áreas localizadas lejos de una clínica y en áreas rurales lejanas a la ciudad de Guatemala, está relacionada con la baja consulta a médicos y enfermeras durante el embarazo y la atención del parto. La probabilidad de consulta con una comadrona se incrementa según la distancia a la clínica o la lejanía de la comunidad; sin embargo, el incremento no es monotónico.

Mientras las diferencias étnicas y geográficas en el uso de las inmunizaciones infantiles son sustanciales, no son tan grandes como aquellas para el control prenatal y la atención del parto. Por ejemplo, menos niños indígenas en comparación a los ladinos fueron inmunizados completamente, pero más de tres cuartas partes de los niños indígenas han recibido al menos una vacuna, y un porcentaje sustancial ha recibido las ocho vacunas. Los números indican que el gobierno, los médicos voluntarios o privados y las enfermeras fueron más exitosos en llegar a la población indígena y a aquellos que viven en las áreas más remotas, por medio del programa de inmunización, que por medio de los servicios de control prenatal y de parto.

Es mucho menos probable tener contactos múltiples con los servicios biomédicos de salud entre las indígenas que no hablan español en comparación con las que hablan español (véase el cuadro 2). De hecho, ninguna indígena que no habla español recibió todos los tres tipos de servicios de salud (resultados no mostrados). El coeficiente de correlación indica que consultar biomédicos para el control prenatal y para la atención del parto son hechos fuertemente relacionados el uno con el otro entre mujeres indígenas que hablan español y ladinas. Por otra parte, la correlación entre servicios biomédicos relacionados con el embarazo e inmunización total es relativamente baja entre los tres grupos etnolingüísticos.

La primera serie de razones de chanzas en el cuadro 3 indica que en la ausencia de variables de control, cada uno de los factores étnicos y geográficos están significativamente relacionados con la probabilidad de recibir atención biomédica concerniente con el embarazo y la serie completa de inmunizaciones. En particular, las chanzas de recibir cuidados biomédicos son bajísimas para las 
indígenas que no hablan español en comparación con las mujeres ladinas (1:20 para asistencia biomédica del parto). Las mujeres que viven en los municipios predominantemente indígenas tienen también chanzas muy bajas de usar servicios biomédicos en comparación a aquellas que viven en municipios ladinos (menos del 1:5 de cuidados biomédicos relacionados con el embarazo). Además, las chanzas de recibir cuidados de salud biomédicos son relativamente bajas en comunidades localizadas lejos de una clínica y en áreas rurales alejadas de la ciudad de Guatemala.

Cuando se controlan las características del infante y de la familia en el modelo, las diferenciales en el uso para las variables étnicas y de localidad de residencia se ven reducidas (o sea que las razones de chanzas son mayores que en el modelo sin controles). No obstante, las chanzas relativas de recibir atención relacionada con el embarazo por parte de médicos o enfermeras se mantienen muy bajas entre ciertos grupos, en particular los indígenas que no hablan español, las mujeres que viven en comunidades predominantemente indígenas y mujeres que viven en comunidades relativamente remotas de una clínica. Por otra parte, una vez que las características del infante y de la familia se mantienen constantes, la lejanía de la comunidad no está significativamente relacionada con el uso de los servicios de la salud.

Las razones de chanzas indican que, con sólo una excepción, las variables étnicas y geográficas no están significativamente relacionadas con la inmunización. En particular, aun cuando es mucho menos probable que la atención biomédica relacionada con el embarazo sea recibida por mujeres indígenas en comparación con mujeres ladinas, ambos grupos tienen la misma probabilidad de inmunizar a sus hijos. Del mismo modo, los resultados indican que los niveles de educación de la mujer y de su esposo están positivamente relacionados con la chanza de recibir servicios biomédicos relacionados con el embarazo (resultados no mostrados), pero están poco relacionados (y probablemente no lo estén) con la probabilidad de recibir una serie completa de inmunizaciones $(\mathrm{Pe}-$ bley y Goldman, 1992). La única relación significativa con las inmunizaciones es la baja probabilidad para los infantes que viven en comunidades predominantemente indígenas.

\section{Discusión}

Los resultados muestran que los programas de salud basados predominantemente en campañas alcanzan con frecuencia a más sectores de la población en comparación con los servicios provis- 
tos por clínicas. En Guatemala, a finales de los años ochenta, la inmunización infantil estaba mucho más extendida y menos relacionada con factores socioeconómicos y étnicos en comparación con la utilización de servicios de médicos y enfermeras para el control prenatal y la atención del parto. Aunque no existe información específica sobre si las inmunizaciones fueron recibidas durante la campaña, el patrón general de uso sugiere que la campaña de 1986 fue efectiva en alcanzar áreas y poblaciones que no habían sido cubiertas por los servicios de algún centro médico.

El ejemplo de Guatemala en los años ochenta destaca varias diferencias importantes entre las campañas de inmunización infantil y los servicios relacionados con el embarazo, las cuales resumimos a continuación:

1. A diferencia de las campañas de inmunización, los servicios relacionados con el embarazo tienen que ser proporcionados por proveedores que se encuentren en la comunidad o cerca de la comunidad a toda hora. A pesar de los esfuerzos del gobierno de Guatemala por extender los servicios de salud en áreas rurales marginadas desde principios de los años setenta, la disponibilidad de servicios basados en clínicas y traslados de emergencias a hospitales aún varían considerablemente. Aunque los servicios de las clínicas sean accesibles, muchas clínicas tienen horarios muy limitados, poco personal y falta de abastecimientos.

2. Puesto que el control prenatal y la atención del parto requieren más contacto entre pacientes y proveedores de servicios, la clase social y las diferencias étnicas entre proveedores y pacientes son muy importantes e influyen considerablemente más que en el caso de las campañas de inmunización. En comunidades indígenas guatemaltecas, por ejemplo, comunicarse con pacientes puede ser difícil porque el personal de la clínica rara vez habla otro idioma que el español (Bosser y Del Cid Peralta, 1987). Además, los pacientes indígenas son motivo de un constante trato discriminatorio y son regularmente menospreciados por comentarios que hace el personal de la clínica (Rosenthal, 1987), que por lo general es ladino.

3. Las creencias tradicionales sobre la salud pueden tener un papel más importante en la selección de servicios relacionados con el embarazo para una mujer en comparación con su selección sobre la inmunización de sus hijos. Las inmunizaciones están muy relacionadas con la medicina biomédica y, por lo general, no se encuentran a la disposición de los proveedores. El control prenatal y la atención del parto, por otro lado, ocupan un importante lugar en las creencias y prácticas tradiciona- 
les de salud. Como en otras partes de Latinoamérica (Pedersen y Barauffati, 1985), creencias tradicionales sobre la fisiología, la causa y el tratamiento de problemas de salud mantienen un sitio predominante tanto para los indígenas como para la población ladina. Así, médicos, enfermeras y comadronas con entrenamiento biomédico son frecuentemente menos aceptados por mujeres embarazadas dado que sus prácticas no corresponden a las creencias de la mujer sobre el embarazo y el parto, no explican procedimientos y tratamientos dentro del contexto de estas creencias y, por lo general, son ajenos a la comunidad y no tienen ninguna conexión social con la mujer y su familia (Quezada et al., 1988).

Debido a las dificultades para la expansión y el mejoramiento de centros médicos, la barrera social entre médicos y pacientes, así como la continua importancia de las creencias tradicionales sobre salud y las parteras tradicionales, es evidente que las mejoras en los servicios relacionados con el embarazo para mujeres guatemaltecas -cuando menos a corto plazo- provendrán principalmente de esfuerzos concentrados en el adiestramiento y el apoyo institucional para comadronas tradicionales (Segeplan, 1991). Esfuerzos previos en el adiestramiento de parteras en Guatemala (Cosminsky, 1982) y México (Jordan, 1989) indican, sin embargo, que los métodos estandarizados para adiestrar parteras tradicionales frecuentemente son inadecuados. Apoyos institucionales y sistemas de referencia de emergencias son también esenciales (Grant, 1993) para que los servicios relacionados con el embarazo sean exitosos durante los años noventa.

\section{Bibliografía}

Bossert, T.J. y E. Del Cid Peralta (1987), Guatemala: Health Sector Assessment, reporte preparado para la U.S. Agency for International Development.

Cosminsky, S. (1982), "Childbirth and Change: A Guatemala Study", en C.P. MacCormack (comp.), Ethnography of Fertility and Birth, Nueva York, Academic Press, pp. 205-229.

Elo, Irma (1990), "Female Education and the Use of Maternal and Child Health Services in Peru", tesis de doctorado, Ann Arbor (MI), Princeton University, University Microfilms.

Gadmoski, A. y R.E. Black (1990), "Impact of Direct Interventions", en K. Hill (comp.), Child Survival Program: Issues of the 1990's, Baltimore, Institute for International Programs, School of Hygiene and Public Health, Johns Hopkins University. 
Goldman, N. y A.R. Pebley (1994), "Health Cards, Maternal Reports, and the Measurement of Immunization Coverage in Guatemala", en Social Science and Medicine, vol. 38, núm. 8, pp. 1075-1089.

Grant, J.P. (1993), The State of the World's Children, Nueva York, Oxford University Press (publicado para Unicef), p. 59.

Hosmer, D.W. y S. Lemeshow (1989), Applied Logistics Regression, Nueva York, John Wiley and Sons.

Huber, P.J. (1967), "The Behavior of Maximum Likelihood Estimates under non-Standard Conditions", en Proceedings of the Fifth Berkeley Symposium on Mathematical Statistics and Probability, núm. 1, pp. 221-223.

Institute of Medicine (1985), Preventing Low Birthweight, Washington D.C., National Academy Press.

Isenalumbe, A.E. (1990), "Integration of Traditional Birth Attendants into Primary Health Care”, en Forum, núm. 6, pp. 69-88.

Jordan, B. (1989), "Cosmopolitical Obstetrics: Some Insights from the Training of Traditional Midwives", en Social Science and Medicine, vol. 28, núm. 9, pp. 925-944.

Kwast, B.E. (1991), "Safe Motherhood: A Challenge to Midwifery Practice", en World Health Forum, núm. 12, pp. 1-6.

Mahler, H. (1987), "The Safe Motherhood Initiative: A Call to Action", en Lancet, núm. 1, pp. 268-270.

Ministerio de Salud Pública y Asistencia Social (MSPAs) (1987), Evaluación: Jornadas Nacionales de Vacunación,1986, República de Guatemala.

—_- Instituto de Nutrición de Centroamérica y Panamá (INCAP) y Demographic and Health Surveys (1989), Encuesta Nacional de Salud Materno Infantil 1987, Guatemala.

Parra, P. (1991), "La mujer rural, las comadronas y el sistema mexicano de salud", en Estudios Demográficos y Urbanos, vol. 6, núm. 1 (16), México, El Colegio de México, pp. 69-99.

Pebley, A.R. (1993), "The Goals of the World Summit for Children and Their Implications for Health Policy in the 1990s", en J. Gribble y S. Preston (comps.), Policy and Planning Implications of the Epidemiological Transition in Developing Countries, Washington D.C., National Academic Press.

__ y N. Goldman (1992), "Family, Community, Ethnic Identity and the Use of Formal Health Care Services in Guatemala", Working Paper 92/12, Princeton, Office of Population Research, Princeton University.

Pedersen, D. y V. Barauffati (1985), "Health and Traditional Medicine Cultures in Latin America and the Caribbean", en Social Science and Medicine, vol. 21, núm. 1, pp. 5-12.

Quezada, R.A., C.H. Peidra, G.V. Delgado, E.D. Aguilar y J.H. Córdova (1988), La práctica médica tradicional, Quito (Ecuador), inıcsA, Universidad de Cuenca, t. 1.

Rosenthal, C. (1987), "Santa María de Jesús: Medical Choice in a Highland Guatemalan Town", tesis no publicada, Cambridge (Mass.), Department of Anthropology, Harvard University, revisado para el Ins- 
tituto de Nutrición de Centroamérica y Panamá.

Secretaría General del Consejo Nacional de Planificación Económica (Segeplan) (1991), Plan de Acción de Desarrollo Social: Desarrollo Humano, Infancia y Juventud 1992-1996, Guatemala.

Von Hoegen, Miguel (1986), Concentración geográfica de servicios en Guatemala,Guatemala, Asociación de Investigación y Estudios Sociales (Monografías, 5).

Warren, C., R.S. Monteith, J.T. Johnson, R. Santiso, F. Guerra y M.W. Oberle (1987), "Use of Maternal-child Health Services and Contraception in Guatemala and Panamá", en Journal of Biosocial Sciences, núm. 19, pp. 299-243.

Warren, K.S. (1988), "The Evolution of Selective Primary Health Care", en Social Science and Medicine, vol. 19, núm. 2, pp. 229-243. 